BULLETIN OF THE

AMERICAN MATHEMATICAL SOCIETY

Volume 77, Number 4, July 1971

\title{
MORE DISTANT THAN THE ANTIPODES
}

\author{
BY J. J. SCHÄFFER ${ }^{1}$
}

Communicated by Victor Klee, February 12, 1971

1. Introduction. Let $X$ be a real normed linear space, and let $\Sigma(X)$ be its unit ball, with the boundary $\partial \Sigma(X)$. If $\operatorname{dim} X \geqq 2, \delta_{X}$ denotes the inner metric of $\partial \Sigma(X)$ induced by the norm (cf. $[1, \S 3]$ ). If no confusion is likely, we write $\Sigma, \partial \Sigma, \delta$. In [1] we introduced and discussed parameters of $X$ based on the metric structure of $\partial \Sigma$; among them are $D(X)=\sup \{\delta(p, q): p, q \in \partial \Sigma\}$, the inner diameter of $\partial \Sigma$, and $M(X)=\sup \{\delta(-p, p): p \in \partial \Sigma\}$, half the perimeter of $\Sigma$. Obviously, $M(X) \leqq D(X)$, and it was conjectured [1, Conjecture 9.1] that $M(X)=D(X)$ in every case, i.e., that "no pair of points of $\partial \Sigma$ is more distant in $\partial \Sigma$ than the most distant antipodes." This equality was shown to hold if $\operatorname{dim} X=2$ or $\operatorname{dim} X=3$ [1, Theorems 5.4, 5.8], if $D(X)=4$ [3], if $X$ is an L-space [4].

In this paper we explode this conjecture by showing that $M(X)=2$, $D(X)=3$ for $X=C_{0}((0,1])$, the space of continuous real-valued functions on $(0,1]$ that tend to 0 at 0 , with the supremum norm. We observe that this failure of the conjecture is "as strong as possible," since $2 D(X) \leqq M(X)+4$ for every normed space $X$ [3, Theorem 1]. The present result is a simple specific instance of the evaluation of $M(X), D(X)$ for many spaces of continuous functions, which will be carried out in a forthcoming paper. It has appeared useful, however, to give a separate account of this very simple example. In addition, Lemma 1 is required for the general theory. The conjecture remains unresolved, and interesting, for spaces of finite dimension greater than three.

We shall use the terminology, notations, and elementary results of $\S \S 1-3$ of [1]. In particular, a subspace of $X$ is a linear manifold in $X$, not necessarily closed, provided with the norm of $X$. If $Y$ is a subspace of $X$, we obviously have

$$
\delta_{Y}(p, q) \geqq \delta(p, q), \quad p, q \in \partial \Sigma(Y) .
$$

Instead of dealing with the space $C_{0}((0,1])$, we prefer, for technical reasons, to consider the space $C_{\pi}([-1,1])$ of odd continuous realvalued functions on $[-1,1]$ with the supremum norm. The two

AMS 1970 subject classifications. Primary 52A40; Secondary 46E15.

Key words and phrases. Inner metric on unit sphere, inner diameter, perimeter.

1 This work was supported in part by NSF Grant GP-19126. 
spaces are obviously congruent, and therefore any metrical property of one implies the same metrical property of the other. In the rest of this paper, $X$ shall always stand for $C_{\pi}([-1,1])$.

2. The perimeter. We consider the special function $u \in X$ defined by $u(t)=t, t \in[-1,1]$.

Lemma 1. $\delta(-u, u)=2$.

PRoOF. For each given integer $n>1$, let $R_{n}=l^{\infty}(\{1, \cdots, n\})$ be the Banach space of sequences of length $n$ of real numbers, with the maximum norm. The proof will depend on the computation of the length of certain polygonal curves in $\partial \Sigma\left(R_{n}\right)$, carried out in [2].

Let $Y_{n}$ be the closed subspace of $X$ consisting of the piecewise linear odd continuous real-valued functions on $[-1,1]$ with "corners" at most at $\pm(2 k-1)(2 n-1)^{-1}, k=1, \cdots, n$. Define the linear mapping $\Phi_{n}: Y_{n} \rightarrow R_{n}$ by $\left(\Phi_{n} f\right)(j)=f\left((2 n-4 j+3)(2 n-1)^{-1}\right), j=1, \cdots$, $n$. Since the mapping $j \mapsto 2 n-4 j+3:\{1, \cdots, n\} \rightarrow\{ \pm(2 k-1)$ : $k=1, \cdots, n\}$ is injective and the image contains exactly one of each pair of opposites, $\Phi_{n}$ is bijective; since a piecewise linear function attains its extrema at "corners," $\Phi_{n}$ is isometric. Hence $\Phi_{n}$ is a congruence.

Now $u \in Y_{n}$; we consider $\Phi_{n} u \in \partial \Sigma\left(R_{n}\right)$ and compute

$$
\left(\Phi_{n} u\right)(j)=(2 n-4 j+3)(2 n-1)^{-1}, \quad j=1, \cdots, n .
$$

On the other hand, we consider $p_{0} \in \partial \Sigma\left(R_{n}\right)$ given by

$$
p_{0}(j)=(n-2 j+1)(n-1)^{-1}, \quad j=1, \cdots, n ;
$$

we know from [2, Lemma 4$]$ that

$$
\delta_{R_{n}}\left(-p_{0}, p_{0}\right) \leqq 2 n(n-1)^{-1}
$$

(in fact, equality holds). Now $\left(\Phi_{n} u\right)(1)=p_{0}(1)=1$, so the straight-line segment with endpoints $\Phi_{n} u, p_{0}$ lies entirely in $\partial \Sigma\left(R_{n}\right)$; therefore, from (2), (3),

$$
\begin{aligned}
\delta\left(p_{0}, \Phi_{n} u\right) & =\left\|\Phi_{n} u-p_{0}\right\| \\
& =2(2 n-1)^{-1}(n-1)^{-1} \max \{j-1: j=1, \cdots, n\} \\
& =2(2 n-1)^{-1} .
\end{aligned}
$$

Since $\Phi_{n}: Y_{n} \rightarrow R_{n}$ is a congruence, (1), (4), (5) yield

$$
\begin{aligned}
2 & =\|u-(-u)\| \leqq \delta(-u, u) \leqq \delta_{Y_{n}}(-u, u)=\delta_{R_{n}}\left(-\Phi_{n} u, \Phi_{n} u\right) \\
& \leqq \delta_{R_{n}}\left(-\Phi_{n} u,-p_{0}\right)+\delta_{R_{n}}\left(-p_{0}, p_{0}\right)+\delta_{R_{n}}\left(p_{0}, \Phi_{n} u\right) \\
& \leqq 4(2 n-1)^{-1}+2 n(n-1)^{-1}=2+2(4 n-3)(n-1)^{-1}(2 n-1)^{-1} .
\end{aligned}
$$


The integer $n$ was arbitrarily great; we conclude that $\delta(u,-u)=2$.

Theorem 2. For every $f \in \partial \Sigma, \delta(-f, f)=2$. Consequently, $M(X)=2$.

Proof. Since $[-1,1]$ is connected and $f$ is odd, we have $f([-1,1])$ $=[-1,1]$. Since the composition of odd functions is odd, we conclude that the linear mapping $g \mapsto g \circ f: X \rightarrow X$ is isometric, hence a congruence of $X$ onto a subspace $Y$ of $X$. Now $( \pm u) \circ f= \pm f \in Y$; by Lemma 1 and (1) we therefore have

$$
2 \leqq \delta(-f, f) \leqq \delta_{Y}(-f, f)=\delta_{Y}(-u \circ f, u \circ f)=\delta(-u, u)=2 .
$$

\section{The inner diameter.}

Lemma 3. Define $v, w \in \partial \Sigma$ by

$$
\begin{array}{rlrl}
v(t) & =-v(-t)=t-\frac{1}{2}+\left|t-\frac{1}{2}\right|, & & 0 \leqq t \leqq 1, \\
w(t) & =-w(-t)=-t-\frac{1}{2}+\left|t-\frac{1}{2}\right|, & 0 \leqq t \leqq 1 .
\end{array}
$$

Then $\delta(v, w) \geqq 3$.

Proof. Let $\mathrm{c}$ be any curve from $v$ to $w$ in $\partial \Sigma$, and $r$ a given number, $0 \leqq r<1$. Since $\|v-v\|=0,\|v-w\|=2$, there exists a point $z$ on $c$ such that $\|z-v\|=r$. Since $z \in \partial \Sigma$ there exists $t \in[-1,1]$ such that $z(t)=1$. Now $v(t) \geqq z(t)-\|z-v\|=1-r>0$. From the definition of $v$ and $w$ we have $t>\frac{1}{2}$, whence $w(t)=-1$. Then

$$
l(\mathfrak{c}) \geqq\|w-z\|+\|z-v\| \geqq|w(t)-z(t)|+r=2+r .
$$

Since $r$ was arbitrarily close to 1 , we have $l(\mathfrak{c}) \geqq 3$. Since $\mathfrak{c}$ was an arbitrary curve from $v$ to $w$ in $\partial \Sigma$, we indeed have $\delta(v, w) \geqq 3$.

REMARK. It is easy to show directly that $\delta(v, w)=3$; there exists, in fact, a curve from $v$ to $w$ in $\partial \Sigma$ consisting of two straight line segments end-to-end, of respective lengths 1 and 2: the intermediate endpoint is $z \in \partial \Sigma$ defined by

$$
z(t)=-z(-t)=t-\frac{3}{2}+3\left|t-\frac{1}{2}\right|, \quad 0 \leqq t \leqq 1 .
$$

The verification is left to the reader.

Theorem 4. $D(X)=3$.

Proof. By [3, Theorem 1], $2 D(X) \leqq M(X)+4$; since $M(X)=2$ by Theorem 2, we conclude, using Lemma 3 , that

$$
3 \leqq \delta(v, w) \leqq D(X) \leqq \frac{1}{2}(2+4)=3,
$$

so that equality holds. 


\section{REFERENCES}

1. J. J. Schäffer, Inner diameter, perimeter, and girth of spheres, Math. Ann. 173 (1967), 59-79. MR $36 \# 1959$.

2. - Addendum: Inner diameter, perimeter, and girth of spheres, Math. Ann. 173 (1967), 79-82. MR 36 \#1959.

3. - - Spheres with maximum inner diameter, Math. Ann. 190 (1971), 242-247.

4. - On the geometry of spheres in L-spaces, Israel J. Math. (to appear).

Carnegie-Mellon University, Pittsburgh, Pennsylvania 15213 